University of Windsor

Scholarship at UWindsor

7-28-2016

\title{
Selective detection of singlet gerade metastable states of $\mathrm{N}-2$
}

Wladyslaw Kedzierski

University of Windsor

J. W. Mcconkey

University of Windsor

Follow this and additional works at: https://scholar.uwindsor.ca/physicspub

Part of the Physics Commons

\section{Recommended Citation}

Kedzierski, Wladyslaw and Mcconkey, J. W.. (2016). Selective detection of singlet gerade metastable states of N-2. JOURNAL OF CHEMICAL PHYSICS, 145 (4), 044313-1-044313-4.

https://scholar.uwindsor.ca/physicspub/188

This Article is brought to you for free and open access by the Department of Physics at Scholarship at UWindsor. It has been accepted for inclusion in Physics Publications by an authorized administrator of Scholarship at UWindsor. For more information, please contact scholarship@uwindsor.ca. 


\title{
Selective Detection of the $\mathrm{a}^{1} \pi_{\mathrm{g}}$ and ${ }^{1} \Sigma_{\mathrm{g}}{ }^{+}$Metastable States of $\mathrm{N}_{2}$.
}

\author{
W Kedzierski and J W McConkey \\ Physics Department, University of Windsor, ON N9B 3P4, Canada.
}

\begin{abstract}
:
Metastable $\mathrm{N}_{2}$ molecules produced by electron impact on $\mathrm{N}_{2}$ are detected using a unique solid nitrogen matrix detector. The time-of-flight (TOF) system is shown to be selectively sensitive to $\mathrm{a}^{1} \pi_{\mathrm{g}}$ and ${ }^{1} \Sigma_{\mathrm{g}}{ }^{+}$metastable species. The latter species had been identified theoretically previously but was detected experimentally for the first time. Their identification and excitation as a function of electron energy from threshold to $300 \mathrm{eV}$ are presented. Comparison is made with data obtained by other techniques.
\end{abstract}

\section{Introduction:}

Metastable atoms and molecules are important species in a wide variety of applications ranging from low temperature electrical discharges to terrestrial and planetary atmospheres and astrophysical sources. Long-lived nitrogen species are intrinsically important because of the dominance of this molecule terrestrially. Many different techniques have been used to study these particles over the years ranging from optical emission or absorption to Auger emission from low workfunction surfaces and Rydberg spectroscopy. Many of these have been referenced and reviewed in the seminal works of Loftus and Krupenie ${ }^{1}$ and Huber and Herzberg $^{2}$. In the present work we present a new technique which is selectively sensitive to certain types of metastables and thus enables studies which are not possible using other more conventional methods. In particular we present evidence for a ${ }^{1} \Sigma_{\mathrm{g}}{ }^{+}$state which, to our knowledge, had not previously been observed.

\section{Experimental Details:}

The apparatus used in this work has been described fully in earlier communications $^{3-5}$ and so only a brief summary will be given together with any details which are particularly relevant to the present work. Full details of the 
experimental set-up and its performance with respect to the use of solid nitrogen matrices for metastable detection has been given elsewhere ${ }^{6}$.

A crossed-beam set-up is used in which a target beam of $\mathrm{N}_{2}$ intersects a pulsed electron beam at right angles. Neutral metastable particles produced drift into a separate chamber and impinge on a cold finger cooled to $10 \mathrm{~K}$ and on which a layer of solid nitrogen has been deposited. The metastables interact with the matrix and release photons which are detected by a photomultiplier. These photons are processed by standard electronics and provide the "Stop" signal for a time-to amplifier convertor (TAC) system Photons produced in coincidence with the electron pulses are reflected off the solid detector surface into the photomultiplier and serve as the "Start" pulses for the detection electronics. Repeated electron pulses lead to the build-up of a time-of-flight (TOF) spectrum. An example of this is shown in Figure 1. As is evident a sharp peak is obtained at very short times due to photons produced during the electron beam pulse and reflected into the detector. A secondary peak at later times marks the arrival of metastables at the detector surface. Suitable gating allows the prompt photon and metastable signals to be monitored separately. Where metastables are produced with just thermal energies, as in the present case, the gas beam direction is pointed towards the detector. Otherwise the initial thermal motion would prevent the metastables from reaching the detector. Currently a plexiglass lightpipe is used to guide photons from the cold finger to the photomultiplier. Filters can be interposed in the photon flight path to limit the wavelength range detected. We note that the metastables pass through a pair of quenching plates en route to the matrix surface. These enable checks to be made for the possible presence of Rydberg species. Charged particles such as ions or scattered electrons are prevented from leaving the gun region and reaching the detector by the 200 gauss magnetic field which is used to confine and direct the electron beam. Prior to operation, a surface layer must be deposited on the surface of the cold finger. During operation this is refreshed continually from the background gas in the system. The cold finger was normally maintained at a temperature of $10 \mathrm{~K}$, the lowest temperature attainable using our He compressor. Calibration of the electron beam energy was achieved by examining the threshold for prompt photon production. For example a $430 \mathrm{~nm}$ filter was used to isolate the $(0,1)$ vibrational $\mathrm{N}_{2}{ }^{+}(\mathrm{B}-\mathrm{X})$ emission at $427.8 \mathrm{~nm}$ with a threshold of $18.8 \mathrm{eV}$.

\section{Results and Discussion.}

Flight times, Figure 1, for the metastables being detected were much longer than when using rare gas matrices to detect metastable atomic species ${ }^{3}$ and, in fact were entirely consistent with the detection of metastable nitrogen molecules rather than atoms. The solid line through the "green" TOF data is a calculated curve assuming 
a gas beam temperature of $292 \mathrm{~K}$ and that the metastables were molecular $\mathrm{N}_{2}$ in nature. The excellent agreement between the observed and calculated curves confirms the identification. To check whether Rydberg species were contributing to the signal we applied a $5 \mathrm{kV} / \mathrm{cm}$ field between the quenching plates flanking the metastable flight path. No indication of any Rydberg component in the signal was observed. This is consistent with the results of Tarr et $\mathrm{al}^{7}$ who investigated $\mathrm{N}_{2}$ high Rydberg production under electron impact and found the cross section to be very small and only significant very close to threshold.

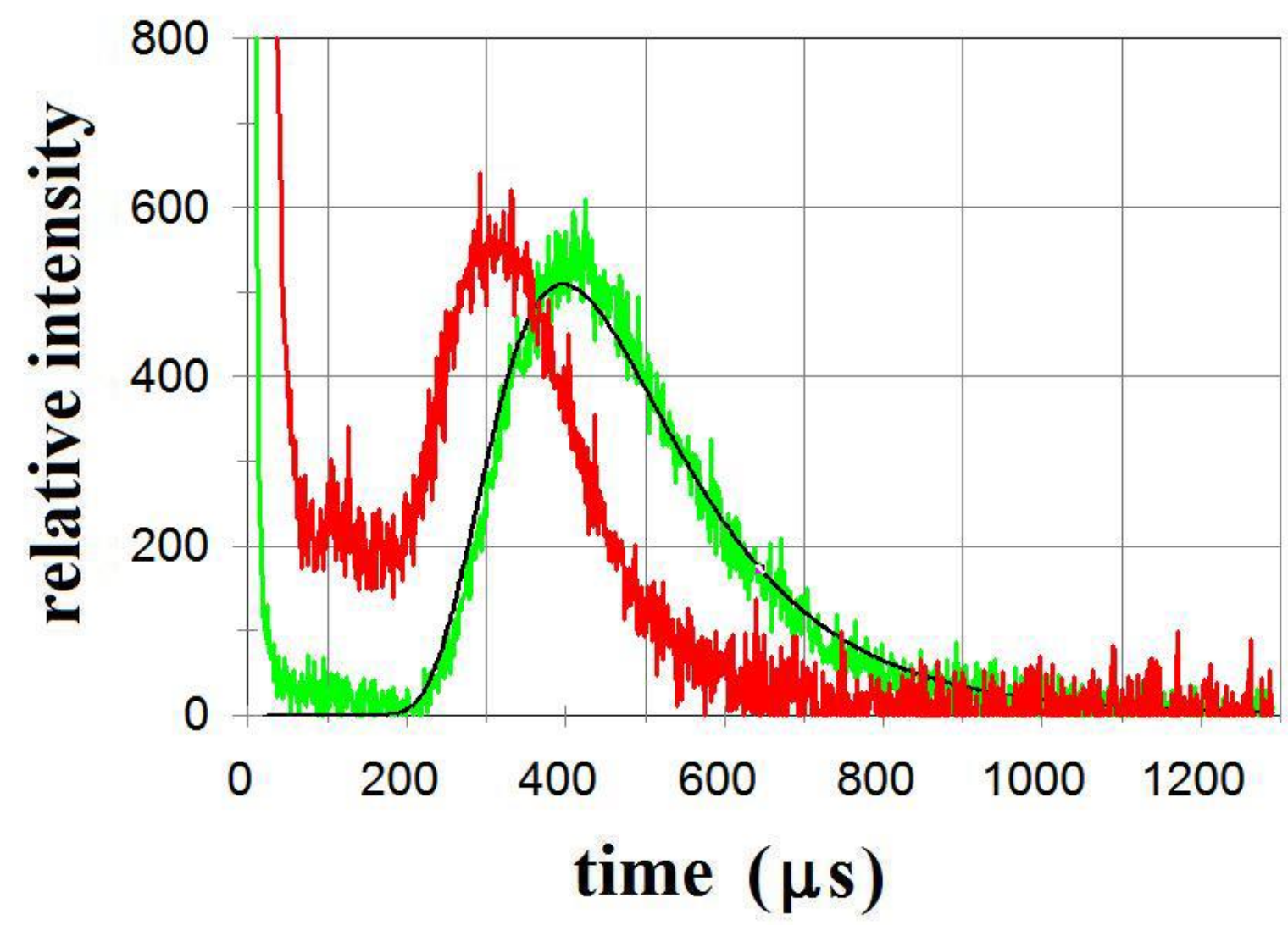

Figure 1. TOF data obtained using red and green filters in the detection channel. In both cases the energy of the pulsed electron beam was $100 \mathrm{eV}$. The steep peak at very short flight times is due to prompt photons emitted during the electron beam pulse and reflected into the photomultiplier from the cold finger. The solid curve through the "green" data is a calculated curve assuming a gas beam temperature of 292 K. See text for further details. 
The distinct differences in the data obtained with red and green filters indicate that different metastable species were being observed. The fact that the "red" peak was observed at shorter TOFs is due to the loss of metastables en route to the detector via in-flight decay resulting in a truncation of the TOF curve at longer flight times. Assuming this to be the case the lifetime of the "red" component was estimated to be $\sim 75 \mu$ s. This lifetime will be a lower limit as there is also the possibility of some loss via collisions en route to the detector. The $\mathrm{a}^{1} \pi_{\mathrm{g}}$ state of $\mathrm{N}_{2}$ has a reported lifetime in this region (see Reference 1 for fuller details) so it might well be the metastable responsible for the red emission from the matrix. Evidence presented below confirms this designation.

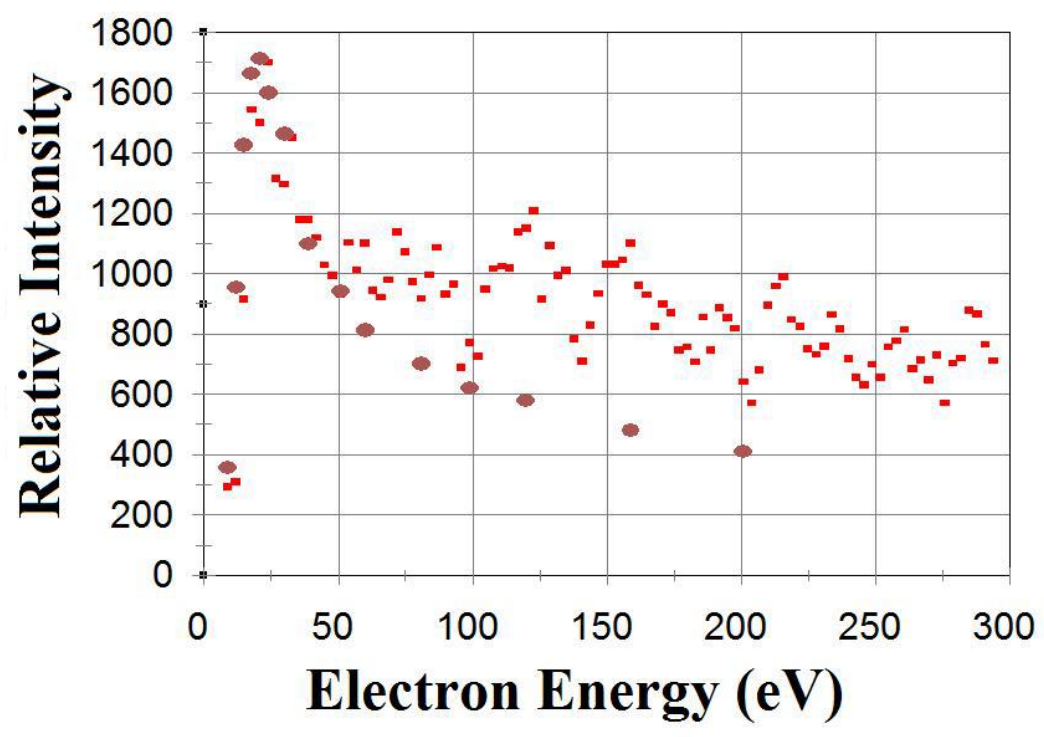

Figure 2. Variation of the metastable signal with impact electron energy when using the red filter in the detection channel. Solid squares - present data; ovals $\mathrm{a}^{1} \pi_{\mathrm{g}}$ excitation data of Young et $\mathrm{al}^{8}$. The two data sets have been normalized at the peak and some smoothing has been applied to the present data at larger energies. A large background signal, arising from scattered light from the filament of the electron gun, and which does not vary with electron energy, has been subtracted from our data.

Figure 2 shows the variation of metastable excitation probability with exciting electron energy when using the red filter in the detection channel. A large constant 
background signal has been subtracted from the data. This was due to stray light from the filament of the electron gun and did not vary with electron energy.

We note a sharp rise from a threshold at approximately $9 \mathrm{eV}$ to a peak some $10 \mathrm{eV}$ higher followed by a fall-off to higher energies. We note that this curve is very similar in both threshold and shape to the one obtained from electron excitation measurements ${ }^{8}$ of the $\mathrm{a}^{1} \pi_{\mathrm{g}}$ state also shown in Figure 2 . The Young et $\mathrm{al}^{8}$ measurements were emission cross section measurements but they demonstrate good agreement in the shape of the excitation probability curve with earlier integral cross section data obtained from inelastic electron scattering data ${ }^{9-11}$ and from direct metastable detection using a channeltron electron multiplier ${ }^{12}$.

Given that our lifetime determination of this metastable state also suggested that it could be the $\mathrm{a}^{1} \pi_{\mathrm{g}}$ state, these measurements would seem to confirm this identification. Since the most likely system which is being emitted in the red is the $\mathrm{N}_{2}$ First Positive (B - A) System we suggest that the following energy transfer and emission processes occur when the $\mathrm{a}^{1} \pi$ molecule arrives at the solid $\mathrm{N}_{2}$ matrix:

$$
\mathrm{N}_{2}\left(\mathrm{a}^{1} \pi_{\mathrm{g}}, \mathrm{v}\right) \rightarrow \mathrm{N}_{2}\left(\mathrm{~B}^{3} \Pi_{\mathrm{g}}, \mathrm{v}^{\prime}\right) \rightarrow \mathrm{N}_{2}\left(\mathrm{~A}^{3} \Sigma_{\mathrm{u}}, \mathrm{v}^{\prime \prime}\right)+\mathrm{h} \gamma(\mathrm{red})
$$

A completely different picture is obtained when the green filter is used in the detection channel. This is illustrated in Figure 3. The metastable signal is observed to rise from a threshold around $12 \mathrm{eV}$ to a broad maximum between 50 and $100 \mathrm{eV}$ followed by a slow fall off towards higher energies. We note that this excitation probability curve is very different to that observed by for example Borst and Zipf ${ }^{13}$ using a more conventional Auger emission detector. They observe the $\mathrm{A}\left({ }^{3} \Sigma_{\mathrm{u}}{ }^{+}\right)$, $\mathrm{E}\left({ }^{3} \Sigma_{\mathrm{g}}{ }^{+}\right)$and a $\left({ }^{1} \pi_{\mathrm{g}}\right)$ states with a combined excitation function which peaks close to $15 \mathrm{eV}$ and falls off rapidly towards higher energies.

What happens when this metastable molecule reaches the matrix is not clear. Two possibilities can be suggested:

In the first, transfer could take place from the metastable state to a dissociating state resulting in the formation of an $\mathrm{N}\left({ }^{2} \mathrm{D}^{0}\right)$ metastable atom. This could form an excimer with another $\mathrm{N}_{2}$ molecule resulting in emission near $523 \mathrm{~nm}$ [see reference 14 and included references]. The threshold energy for this process is at least 12.1 $\mathrm{eV}$ (the dissociation limit for producing $\mathrm{N}\left({ }^{4} \mathrm{~S}\right)+\mathrm{N}\left({ }^{2} \mathrm{D}^{0}\right)$ ), in agreement with our measurements. However, use of a $520 \mathrm{~nm}$ filter with a $10 \mathrm{~nm}$ bandwidth in the detection channel showed this possibility to be small; approximately $10 \%$ of the 
total green emission following $100 \mathrm{eV}$ initial excitation occurred in this wavelength region.

In the second, an energy transfer could occur to the $\mathrm{H}^{3} \Phi_{\mathrm{u}}$ or $\mathrm{B}^{3} \Pi_{\mathrm{g}}$ states that are the only $\mathrm{N}_{2}$ states known to give rise to green features ${ }^{1,15}$, the Gaydon-Herman and First Positive bands respectively. The former are the more likely candidates since the B state dissociation limit is $12.1 \mathrm{eV}$ and so the processes involved may be indicated by:

$\mathrm{e}+\mathrm{N}_{2}\left(\mathrm{X}^{1} \Sigma_{\mathrm{g}}{ }^{+}\right) \rightarrow \mathrm{N}_{2}{ }^{\mathrm{m}}(\mathrm{v}) \rightarrow \mathrm{N}_{2}\left(\mathrm{H}^{3} \Phi_{\mathrm{u}}, \mathrm{v}^{\prime}\right) \rightarrow \mathrm{N}_{2}\left(\mathrm{G}^{3} \Delta_{\mathrm{g}}, \mathrm{v}^{\prime /}\right)+\mathrm{h} \gamma($ green $)$

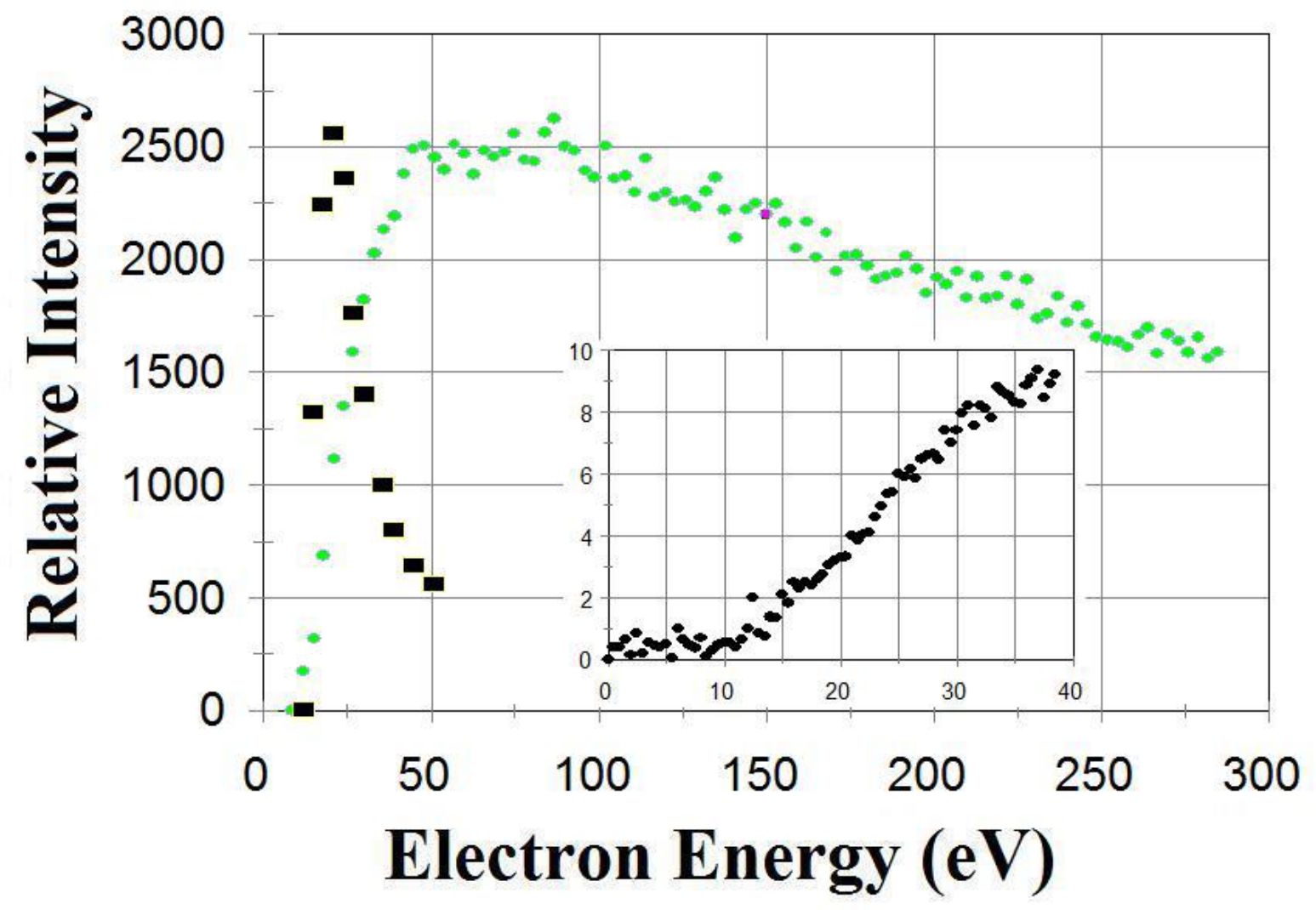

Figure 3. Metastable signal as a function of impact electron energy when using a green filter in the detection channel. A constant background signal has been subtracted from the data. The inset gives additional data showing the nearthreshold region in more detail. The solid rectangles refer to recommended cross section values of Itikawa ${ }^{16}$ for excitation of the a ${ }^{1} \Sigma_{\mathrm{g}}{ }^{+}$metastable state which have been normalized to the present data at the peak. 
A possible candidate for the $\mathrm{N}_{2}$ metastable state is the a" $\left({ }^{1} \Sigma_{\mathrm{g}}{ }^{+}\right)$. Electric dipole selection rules do not allow this state to decay radiatively to any known state of lower energy. In fact the state was identified in early electron energy loss studies ${ }^{17,18}$ as being linked to the ground state by an electric quadrupole transition. The broad maximum in the excitation probability curve (Figure 3 ) suggests that the initial excitation in the parent molecule is to a spin allowed but optically forbidden, i.e. singlet gerade, state. Furthermore, the measured onset energy for excitation $(12.0-13.0 \mathrm{eV})$ is consistent with the energy $(12.26 \mathrm{eV})$ of the $\mathrm{a}^{1}{ }^{1} \Sigma_{\mathrm{g}}{ }^{+}$state within the error of our measurements.

However this identification of our metastable state with a" ${ }^{1} \Sigma_{\mathrm{g}}{ }^{+}$can be discounted given the following facts. First, our TOF data, Figure 1, suggests a lifetime much longer than $500 \mu$ s whereas Kam and Pipkin ${ }^{19}$ have measured the lifetime of the $\mathrm{v}=0$ vibrational level to be a mere $3.5 \mu$ s. They suggested that this was due to predissociation of this level. Second, Itakawa ${ }^{16}$ has discussed a number of evaluations of the a" ${ }^{1} \Sigma_{\mathrm{g}}{ }^{+}$excitation probability curve and presents the recommended data set also shown in Figure 3. Clearly there is a wide divergence between this curve and our measured data. This leads us to the conclusion that our data represent the first observation of the other ${ }^{1} \Sigma_{\mathrm{g}}{ }^{+}$state in this $12 \mathrm{eV}$ energy region that was identified by Michels ${ }^{20}$ in his ab initio configuration interaction

calculations. This state has a deep minimum at an inter-nuclear separation of $1.6 \AA$. Since this is much larger than the ground state minimum of $1.2 \AA$, one would expect based on the Franck-Condon Principle that it would be only weakly excited in electron collisions.

\section{Conclusions.}

Evidence is presented for the excitation and detection of metastable $\mathrm{N}_{2}$ molecules using a novel solid nitrogen matrix detector. The states detected were a ${ }^{1} \Sigma_{\mathrm{g}}{ }^{+}$state, which had been identified earlier in theoretical calculations by Michels ${ }^{20}$ but had not been observed previously, and a $\mathrm{a}^{1} \pi_{\mathrm{g}}$ state whose properties are well known. Data are presented over an electron energy range from threshold to $300 \mathrm{eV}$. The matrix detector was insensitive to other $\mathrm{N}_{2}$ metastable triplet states such as $\mathrm{A}\left({ }^{3} \Sigma_{\mathrm{u}}{ }^{+}\right)$ and $\mathrm{E}\left({ }^{3} \Sigma_{\mathrm{g}}{ }^{+}\right)$. 


\section{Acknowledgements.}

The authors gratefully acknowledge financial support from NSERC (Canada) and CFI (Canada) and invaluable technical support from the University of Windsor, Physics Department, mechanical and electronic workshops. F Alsaiari helped with some of the initial data processing.

\section{References:}

1. A Loftus and P H Krupenie, J Phys Chem Ref Data,6, 113, (1977).

2. K P Huber and G Herzberg, "Molecular Spectra and Molecular Structure, IV. Constants of Diatomic Molecules", Van Nostrand Reinold Co, New York, (1979).

3. J W McConkey and W Kedzierski, Adv At Mol Opt Phys, 63, 1, (2014).

4. W Kedzierski, J Borbely and J W McConkey, J Phys B, 34, 4027, (2001).

5. L R LeClair and J W McConkey, J Phys B, 27, 4039, (1994).

6. W Kedzierski and J W McConkey, Rev Scient Instr. In Press, (2016).

7. S M Tarr, J A Schiavone, and R S Freund, J Chem Phys, 74, 2869, (1981).

8. J A Young, C P Malone, P V Johnson, J M Ajello, X Liu and

I Kanik, J Phys B, 43, 135201, (2010).

9. P V Johnson, C P Malone, I Kanik, K Tran and M A Khakoo, J Geophys Res, 110, A11311, (2005).

10. L Campbell, M J Brunger, A M Nolan, L J Kelly, A B Wedding, J Harrison, P J O Teubner, D C Cartwright and B McLaughlin, J Phys B, 34, 1185, (2001).

11. S Trajmar, D F Register and A Chutjian, Phys Rev, 97, 219, (1983).

12. N J Mason and W R Newell, J Phys B, 20, 3913, (1987).

13. W L Borst and E C Zipf, Phys Rev A, 3, 979, (1971).

14. O Oehler, D A Smith and K Dressler, J Chem Phys, 66, 2097, (1977).

15. P K Carroll, C C Collins and J T Murnahan, J Phys B, 5, 1634, (1972).

16. Y Itikawa,

17. H G M Heideman, C E Kuyatt and G E Chamberlain, J Chem Phys, 44, 355, (1966).

18. E N Lassettre, A Skerbele and V D Meyer, J Chem Phys, 45, 3214, (1966).

19. A W Kam and F M Pipkin, Phys Rev A, 43, 3279, (1991).

20. H Michels, J Chem Phys, 53, 841, (1970). 